\title{
Beam Energy and Centrality Dependence of Direct-Photon Emission from Ultrarelativistic Heavy-Ion Collisions
}

A. Adare, ${ }^{14}$ S. Afanasiev,${ }^{34}$ C. Aidala,${ }^{15,44,49,50}$ N. N. Ajitanand, ${ }^{70, *}$ Y. Akiba, ${ }^{64,65, \dagger}$ R. Akimoto,${ }^{13}$ H. Al-Bataineh,${ }^{58}$ J. Alexander, ${ }^{70}$ M. Alfred, ${ }^{27}$ A. Al-Jamel, ${ }^{58}$ H. Al-Ta'ani, ${ }^{58}$ A. Angerami, ${ }^{15}$ K. Aoki, ${ }^{37,40,64}$ N. Apadula,,${ }^{32,71}$ L. Aphecetche, ${ }^{72}$ Y. Aramaki, ${ }^{13,64}$ R. Armendariz, ${ }^{58}$ S. H. Aronson, ${ }^{8}$ J. Asai, ${ }^{64,65}$ H. Asano, ${ }^{40,64}$ E. C. Aschenauer ${ }^{8}$ E. T. Atomssa, ${ }^{41,71}$ R. Averbeck, ${ }^{71}$ T. C. Awes, ${ }^{60}$ B. Azmoun, ${ }^{8}$ V. Babintsev, ${ }^{28}$ A. Bagoly, ${ }^{19}$ M. Bai, ${ }^{7}$ G. Baksay, ${ }^{22}$ L. Baksay, ${ }^{22}$ A. Baldisseri, ${ }^{17}$ B. Bannier, ${ }^{71}$ K. N. Barish, ${ }^{9}$ P. D. Barnes, ${ }^{44, *}$ B. Bassalleck, ${ }^{57}$ A. T. Basye, ${ }^{1}$ S. Bathe, ${ }^{6,9,65}$ S. Batsouli, ${ }^{15,60}$ V. Baublis, ${ }^{63}$ F. Bauer, ${ }^{9}$ C. Baumann, ${ }^{51}$ S. Baumgart, ${ }^{64}$ A. Bazilevsky, ${ }^{8}$ S. Belikov, ${ }^{8,32, *}$ R. Belmont, ${ }^{14,77}$ R. Bennett, ${ }^{71}$ A. Berdnikov, ${ }^{67}$ Y. Berdnikov, ${ }^{67}$ J. H. Bhom, ${ }^{81}$ A. A. Bickley, ${ }^{14}$ M. T. Bjorndal, ${ }^{15}$ D. S. Blau, ${ }^{39,56}$ M. Boer,${ }^{44}$ J. G. Boissevain, ${ }^{44}$ J. S. Bok, ${ }^{57,58,81}$ H. Borel, ${ }^{17}$ K. Boyle,${ }^{65,71}$ M. L. Brooks, ${ }^{44}$ D. S. Brown, ${ }^{58}$ J. Bryslawskyj, ${ }^{6,9}$ D. Bucher, ${ }^{51}$ H. Buesching, ${ }^{8}$ V. Bumazhnov, ${ }^{28}$ G. Bunce, ${ }^{8,65}$ J. M. Burward-Hoy, ${ }^{44}$ S. Butsyk,${ }^{44,57,71}$ C. M. Camacho, ${ }^{44}$ S. Campbell, ${ }^{15,71}$ V. Canoa Roman, ${ }^{71}$ A. Caringi, ${ }^{52}$ P. Castera, ${ }^{71}$ J.-S. Chai, ${ }^{36,73}$ B. S. Chang, ${ }^{81}$ W. C. Chang, ${ }^{2}$ J.-L. Charvet, ${ }^{17}$ C.-H. Chen, ${ }^{65,71}$ S. Chernichenko, ${ }^{28}$ C. Y. Chi, ${ }^{15}$ J. Chiba,${ }^{37}$ M. Chiu, ${ }^{8,15,29}$ I. J. Choi ${ }^{29,81}$ J. B. Choi, ${ }^{11, *}$ S. Choi, ${ }^{69}$ R. K. Choudhury, ${ }^{5}$ P. Christiansen, ${ }^{46}$ T. Chujo, ${ }^{76,77}$ P. Chung, ${ }^{70}$ A. Churyn, ${ }^{28}$ O. Chvala, ${ }^{9}$ V. Cianciolo, ${ }^{60}$ Z. Citron, ${ }^{71,79}$ C. R. Cleven, ${ }^{24}$ Y. Cobigo, ${ }^{17}$ B. A. Cole, ${ }^{15}$ M. P. Comets, ${ }^{61}$ Z. Conesa del Valle, ${ }^{41}$ M. Connors, ${ }^{24,65,71}$ P. Constantin, ${ }^{32,44}$ M. Csanád, ${ }^{19}$ T. Csörgő, ${ }^{20,80}$ T. Dahms, ${ }^{71}$ S. Dairaku, ${ }^{40,64}$ I. Danchev, ${ }^{77}$ T. W. Danley, ${ }^{59}$ K. Das, ${ }^{23}$ A. Datta, ${ }^{49}$ M. S. Daugherity, ${ }^{1}$ G. David, ${ }^{8,71}$ M. K. Dayananda, ${ }^{24}$ M. B. Deaton, ${ }^{1}$ K. Dehmelt, ${ }^{22,71}$ H. Delagrange, ${ }^{72, *}$ A. Denisov, ${ }^{28}$ D. d'Enterria, ${ }^{15,41}$ A. Deshpande ${ }^{65,71}$ E. J. Desmond, ${ }^{8}$ K. V. Dharmawardane, ${ }^{58}$ O. Dietzsch, ${ }^{68}$ L. Ding, ${ }^{32}$ A. Dion, ${ }^{32,71}$ J. H. Do, ${ }^{81}$ M. Donadelli, ${ }^{68}$ L. D’Orazio, ${ }^{48}$ J. L. Drachenberg, ${ }^{1}$ O. Drapier, ${ }^{41}$ A. Drees,${ }^{71}$ K. A. Drees, ${ }^{7}$ A. K. Dubey, ${ }^{79}$ J. M. Durham,${ }^{44,71}$ A. Durum,${ }^{28}$ D. Dutta, ${ }^{5}$ V. Dzhordzhadze, ${ }^{9,74}$ S. Edwards, ${ }^{7,23}$ Y. V. Efremenko, ${ }^{60}$ J. Egdemir, ${ }^{71}$ F. Ellinghaus, ${ }^{14}$ W. S. Emam, ${ }^{9}$ T. Engelmore, ${ }^{15}$ A. Enokizono, ${ }^{26,43,60,64,66}$ H. En'yo, ${ }^{64,65}$ B. Espagnon, ${ }^{61}$ S. Esumi, ${ }^{76}$ K. O. Eyser, ${ }^{8,9}$ B. Fadem, ${ }^{52}$ W. Fan, ${ }^{71}$ N. Feege, ${ }^{71}$ D. E. Fields, ${ }^{57,65}$ M. Finger, ${ }^{10,34}$ M. Finger, Jr., ${ }^{10,34}$ F. Fleuret, ${ }^{41}$ S. L. Fokin, ${ }^{39}$ B. Forestier, ${ }^{45}$ Z. Fraenkel, ${ }^{79,}{ }^{*}$ J. E. Frantz, ${ }^{15,59,71}$ A. Franz, ${ }^{8}$ A. D. Frawley, ${ }^{23}$ K. Fujiwara ${ }^{64}$ Y. Fukao, ${ }^{40,64}$ S.-Y. Fung, ${ }^{9}$ T. Fusayasu, ${ }^{54}$ S. Gadrat, ${ }^{45}$ K. Gainey, ${ }^{1}$ C. Gal, ${ }^{71}$ P. Gallus, ${ }^{16}$ P. Garg, ${ }^{4,71}$ A. Garishvili, ${ }^{74}$ I. Garishvili, ${ }^{43,74}$ F. Gastineau, ${ }^{72}$ H. Ge, ${ }^{71}$ M. Germain, ${ }^{72}$ A. Glenn, ${ }^{14,43,74}$ H. Gong, ${ }^{71}$ X. Gong, ${ }^{70}$ M. Gonin, ${ }^{41}$ J. Gosset, ${ }^{17}$ Y. Goto,${ }^{64,65}$ R. Granier de Cassagnac, ${ }^{41}$ N. Grau, ${ }^{3,15,32}$ S. V. Greene, ${ }^{77}$ G. Grim, ${ }^{44}$ M. Grosse Perdekamp, ${ }^{29,65}$ T. Gunji, ${ }^{13}$ L. Guo, ${ }^{44}$ H.-Å. Gustafsson, ${ }^{46, *}$ T. Hachiya, ${ }^{26,55,64,65}$ A. Hadj Henni, ${ }^{72}$ C. Haegemann,${ }^{57}$ J. S. Haggerty, ${ }^{8}$ M. N. Hagiwara, ${ }^{1}$ K. I. Hahn, ${ }^{21}$ H. Hamagaki, ${ }^{13}$ J. Hamblen,${ }^{74}$ R. Han,${ }^{62}$ J. Hanks, ${ }^{15,71}$ H. Harada,${ }^{26}$ E. P. Hartouni, ${ }^{43}$ K. Haruna,${ }^{26}$ M. Harvey, ${ }^{8}$ S. Hasegawa, ${ }^{33}$ T. O. S. Haseler, ${ }^{24}$ K. Hashimoto, ${ }^{64,66}$ E. Haslum, ${ }^{46}$ K. Hasuko, ${ }^{64}$ R. Hayano,${ }^{13}$ X. He ${ }^{24}$ M. Heffner, ${ }^{43}$ T. K. Hemmick, ${ }^{71}$ T. Hester, ${ }^{9}$ J. M. Heuser,${ }^{64}$ H. Hiejima, ${ }^{29}$ J. C. Hill, ${ }^{32}$ K. Hill,${ }^{14}$ R. Hobbs, ${ }^{57}$ A. Hodges, ${ }^{24}$ M. Hohlmann, ${ }^{22}$ R. S. Hollis, ${ }^{9}$ M. Holmes ${ }^{77}$ W. Holzmann ${ }^{15,70}$ K. Homma, ${ }^{26}$ B. Hong ${ }^{38}$ T. Horaguchi,,${ }^{13,26,64,75,76}$ Y. Hori, ${ }^{13}$ D. Hornback, ${ }^{74}$ N. Hotvedt, ${ }^{32}$ J. Huang, ${ }^{8}$ S. Huang, ${ }^{77}$ M. G. Hur, ${ }^{36}$ T. Ichihara, ${ }^{64,65}$ R. Ichimiya, ${ }^{64}$ H. Iinuma,${ }^{37,40,64}$ Y. Ikeda, ${ }^{64,76}$

K. Imai ${ }^{33,40,64}$ J. Imrek, ${ }^{18}$ M. Inaba, ${ }^{76}$ Y. Inoue,${ }^{64,66}$ A. Iordanova, ${ }^{9}$ D. Isenhower, ${ }^{1}$ L. Isenhower, ${ }^{1}$ M. Ishihara, ${ }^{64}$

T. Isobe, ${ }^{1,64}$ M. Issah, ${ }^{70,77}$ A. Isupov, ${ }^{34}$ D. Ivanishchev, ${ }^{63}$ Y. Iwanaga, ${ }^{26}$ B. V. Jacak, ${ }^{71}$ M. Javani, ${ }^{24}$ Z. Ji, ${ }^{71}$ J. Jia, ${ }^{8,15,70}$ X. Jiang, ${ }^{44}$ J. Jin, ${ }^{15}$ O. Jinnouchi, ${ }^{65}$ B. M. Johnson, ${ }^{8,24}$ T. Jones, ${ }^{1}$ K. S. Joo, ${ }^{53}$ D. Jouan, ${ }^{61}$ D. S. Jumper, ${ }^{1,29}$ F. Kajihara, ${ }^{13,64}$ S. Kametani, ${ }^{13,64,78}$ N. Kamihara, ${ }^{64,65,75}$ J. Kamin, ${ }^{71}$ M. Kaneta, ${ }^{65}$ S. Kaneti, ${ }^{71}$ B. H. Kang, ${ }^{25}$ J. H. Kang, ${ }^{81}$ J. S. Kang, ${ }^{25}$ H. Kanou, ${ }^{64,75}$ J. Kapustinsky, ${ }^{44}$ K. Karatsu, ${ }^{40,64}$ M. Kasai,${ }^{64,66}$ T. Kawagishi, ${ }^{76}$ D. Kawall, ${ }^{49,65}$ M. Kawashima,${ }^{64,66}$ A. V. Kazantsev, ${ }^{39}$ S. Kelly, ${ }^{14}$ T. Kempel, ${ }^{32}$ V. Khachatryan, ${ }^{71}$ A. Khanzadeev, ${ }^{63}$ K. M. Kijima, ${ }^{26}$ J. Kikuchi, ${ }^{78}$ A. Kim, ${ }^{21}$ B. I. Kim ${ }^{38}$ C. Kim, ${ }^{38}$ D. H. Kim, ${ }^{53}$ D. J. Kim,${ }^{35,81}$ E. Kim, ${ }^{69}$ E.-J. Kim, ${ }^{11}$ H. J. Kim,${ }^{81}$ K.-B. Kim, ${ }^{11}$ M. Kim, ${ }^{69}$ S. H. Kim, ${ }^{81}$ Y.-J. Kim, ${ }^{29}$ Y. K. Kim, ${ }^{25}$ Y.-S. Kim, ${ }^{36}$ D. Kincses, ${ }^{19}$ E. Kinney, ${ }^{14}$ K. Kiriluk, ${ }^{14}$ Á. Kiss, ${ }^{19}$ E. Kistenev, ${ }^{8}$ A. Kiyomichi ${ }^{64}$ J. Klatsky, ${ }^{23}$ J. Klay, ${ }^{43}$ C. Klein-Boesing, ${ }^{51}$ D. Kleinjan, ${ }^{9}$ P. Kline ${ }^{71}$ L. Kochenda ${ }^{63}$ V. Kochetkov ${ }^{28}{ }^{2}$ Y. Komatsu, ${ }^{13,37}$ B. Komkov, ${ }^{63}$ M. Konno, ${ }^{76}$ J. Koster, ${ }^{29}$ D. Kotchetkov, ${ }^{9,59}$ D. Kotov, ${ }^{63,67}$ A. Kozlov, ${ }^{79}$ A. Král, ${ }^{16}$ A. Kravitz, ${ }^{15}$ F. Krizek, ${ }^{35}$ P. J. Kroon, ${ }^{8}$ J. Kubart, ${ }^{10,31}$ G. J. Kunde, ${ }^{44}$ B. Kurgyis, ${ }^{19}$ N. Kurihara, ${ }^{13}$ K. Kurita, ${ }^{64,66}$ M. Kurosawa, ${ }^{64,65}$ M. J. Kweon, ${ }^{38}$ Y. Kwon, ${ }^{74,81}$ G. S. Kyle, ${ }^{58}$ R. Lacey, ${ }^{70}$ Y. S. Lai,${ }^{15}$ J. G. Lajoie, ${ }^{32}$ D. Layton, ${ }^{29}$ A. Lebedev, ${ }^{32}$ Y. Le Bornec,${ }^{61}$ S. Leckey, ${ }^{71}$ B. Lee, ${ }^{25}$ D. M. Lee ${ }^{44}$ J. Lee, ${ }^{21,73}$ K. B. Lee ${ }^{38}$ K. S. Lee, ${ }^{38}$ M. K. Lee, ${ }^{81}$ S. H. Lee ${ }^{32,71}$ S. R. Lee, ${ }^{11}$ T. Lee, ${ }^{69}$ M. J. Leitch, ${ }^{44}$

M. A. L. Leite, ${ }^{68}$ M. Leitgab, ${ }^{29}$ B. Lenzi, ${ }^{68}$ Y. H. Leung, ${ }^{71}$ B. Lewis, ${ }^{71}$ N. A. Lewis, ${ }^{50}$ X. Li,${ }^{12}$ X. Li, ${ }^{44}$ X. H. Li, ${ }^{9}$ P. Lichtenwalner, ${ }^{52}$ P. Liebing, ${ }^{65}$ H. Lim, ${ }^{69}$ S. H. Lim, ${ }^{44,81}$ L. A. Linden Levy, ${ }^{14,29}$ T. Liška, ${ }^{16}$ A. Litvinenko, ${ }^{34}$ H. Liu,,${ }^{44,58}$ M. X. Liu, ${ }^{44}$ S. Lökös, ${ }^{1920}$ B. Love ${ }^{77}$ D. Lynch, ${ }^{8}$ C. F. Maguire, ${ }^{77}$ T. Majoros, ${ }^{18}$ Y. I. Makdisi, ${ }^{7,8}$ M. Makek, ${ }^{79,82}$ 
A. Malakhov ${ }^{34}$ M. D. Malik, ${ }^{57}$ A. Manion, ${ }^{71}$ V. I. Manko, ${ }^{39}$ E. Mannel,${ }^{8,15}$ Y. Mao, ${ }^{62,64}$ L. Mašek, ${ }^{10,31}$ H. Masui ${ }^{76}$ S. Masumoto, ${ }^{13,37}$ F. Matathias, ${ }^{15,71}$ M. C. McCain, ${ }^{29}$ M. McCumber, ${ }^{14,44,71}$ P. L. McGaughey, ${ }^{44}$ D. McGlinchey, ${ }^{14,23,44}$ C. McKinney, ${ }^{29}$ N. Means, ${ }^{71}$ M. Mendoza, ${ }^{9}$ B. Meredith ${ }^{29}$ Y. Miake, ${ }^{76}$ T. Mibe, ${ }^{37}$ A. C. Mignerey, ${ }^{48}$ D. E. Mihalik, ${ }^{71}$ P. Mikeš ${ }^{10,31}$ K. Miki, ${ }^{64,76}$ T. E. Miller ${ }^{77}$ A. Milov,${ }^{8,71,79}$ S. Mioduszewski, ${ }^{8}$ D. K. Mishra, ${ }^{5}$ G. C. Mishra, ${ }^{24}$ M. Mishra, ${ }^{4}$ J. T. Mitchell, ${ }^{8}$ M. Mitrovski, ${ }^{70}$ G. Mitsuka, ${ }^{37,65}$ Y. Miyachi, ${ }^{64,75}$ S. Miyasaka, ${ }^{64,75}$ A. K. Mohanty, ${ }^{5}$ S. Mohapatra, ${ }^{70}$ H. J. Moon, ${ }^{53}$ T. Moon, ${ }^{81}$ Y. Morino, ${ }^{13}$ A. Morreale, ${ }^{9}$ D. P. Morrison, ${ }^{8}$ S. I. Morrow, ${ }^{77}$ J. M. Moss, ${ }^{44}$ S. Motschwiller ${ }^{52}$ T. V. Moukhanova, ${ }^{39}$ D. Mukhopadhyay, ${ }^{77}$ T. Murakami, ${ }^{40,64}$ J. Murata, ${ }^{64,66}$ A. Mwai, ${ }^{70}$ T. Nagae, ${ }^{40}$ S. Nagamiya, ${ }^{37,64}$ K. Nagashima, ${ }^{26}$ Y. Nagata, ${ }^{76}$ J. L. Nagle, ${ }^{14}$ M. Naglis, ${ }^{79}$ M. I. Nagy, ${ }^{19,80}$ I. Nakagawa, ${ }^{64,65}$ Y. Nakamiya, ${ }^{26}$

K. R. Nakamura, ${ }^{40,64}$ T. Nakamura, ${ }^{26,64}$ K. Nakano, ${ }^{64,75}$ S. Nam, ${ }^{21}$ C. Nattrass, ${ }^{74}$ A. Nederlof, ${ }^{52}$ J. Newby, ${ }^{43}$ M. Nguyen, ${ }^{71}$ M. Nihashi, ${ }^{26,64}$ T. Niida, ${ }^{76}$ B. E. Norman, ${ }^{44}$ R. Nouicer, ${ }^{8,65}$ T. Novák, ${ }^{20}$ N. Novitzky, ${ }^{35,71}$ A. S. Nyanin, ${ }^{39}$ J. Nystrand, ${ }^{46}$ C. Oakley ${ }^{24}$ E. O'Brien, ${ }^{8}$ S. X. Oda, ${ }^{13}$ C. A. Ogilvie,${ }^{32}$ H. Ohnishi, ${ }^{64}$ I. D. Ojha,${ }^{77}$ M. Oka, ${ }^{76}$ K. Okada, ${ }^{65}$ O. O. Omiwade, ${ }^{1}$ Y. Onuki, ${ }^{64}$ J. D. Orjuela Koop, ${ }^{14}$ J. D. Osborn, ${ }^{50}$ A. Oskarsson, ${ }^{46}$ I. Otterlund, ${ }^{46}$ M. Ouchida, ${ }^{26,64}$ K. Ozawa, ${ }^{13,37,76}$ R. Pak, ${ }^{8}$ D. Pal, ${ }^{77}$ A. P. T. Palounek, ${ }^{44}$ V. Pantuev, ${ }^{30,71}$ V. Papavassiliou, ${ }^{58}$ B. H. Park, ${ }^{25}$ I. H. Park, ${ }^{21,73}$ J. Park,${ }^{69}$ S. Park,,${ }^{64,69,71}$

S. K. Park, ${ }^{38}$ W. J. Park, ${ }^{38}$ S. F. Pate, ${ }^{58}$ L. Patel, ${ }^{24}$ M. Patel, ${ }^{32}$ H. Pei,${ }^{32}$ J.-C. Peng, ${ }^{29}$ W. Peng, ${ }^{77}$ H. Pereira, ${ }^{17}$

D. V. Perepelitsa, ${ }^{14,15}$ V. Peresedov, ${ }^{34}$ D. Yu. Peressounko, ${ }^{39}$ C. E. PerezLara, ${ }^{71}$ R. Petti,,${ }^{8,71}$ C. Pinkenburg, ${ }^{8}$ R. P. Pisani, ${ }^{8}$ M. Proissl ${ }^{71}$ M. L. Purschke, ${ }^{8}$ A. K. Purwar, ${ }^{44,71}$ H. Qu, ${ }^{1,24}$ P. V. Radzevich, ${ }^{67}$ J. Rak, ${ }^{32,35,57}$ A. Rakotozafindrabe, ${ }^{41}$ I. Ravinovich, ${ }^{79}$ K. F. Read,${ }^{60,74}$ S. Rembeczki, ${ }^{22}$ M. Reuter, ${ }^{71}$ K. Reygers, ${ }^{51}$ D. Reynolds ${ }^{70}$ V. Riabov, ${ }^{56,63}$ Y. Riabov,${ }^{63,67}$ E. Richardson, ${ }^{48}$ D. Richford, ${ }^{6}$ T. Rinn, ${ }^{32}$ D. Roach, ${ }^{77}$ G. Roche, ${ }^{45,}$ S. D. Rolnick, ${ }^{9}$ A. Romana, ${ }^{41,{ }^{*}}$ M. Rosati, ${ }^{32}$ C. A. Rosen, ${ }^{14}$ S. S. E. Rosendahl, ${ }^{46}$ P. Rosnet, ${ }^{45}$ Z. Rowan, ${ }^{6}$ P. Rukoyatkin, ${ }^{34}$ J. Runchey, ${ }^{32}$ P. Ružička, ${ }^{31}$ V. L. Rykov, ${ }^{64}$ S. S. Ryu ${ }^{81}$ B. Sahlmueller, ${ }^{51,71}$ N. Saito, ${ }^{37,40,64,65}$ T. Sakaguchi, ${ }^{8,13,78}$ S. Sakai, ${ }^{76}$ K. Sakashita, ${ }^{64,75}$ H. Sakata, ${ }^{26}$ H. Sako, ${ }^{33}$ V. Samsonov, ${ }^{56,63}$ M. Sano, ${ }^{76}$ S. Sano, ${ }^{13,78}$ M. Sarsour, ${ }^{24}$ H. D. Sato, ${ }^{40,64}$ S. Sato, ${ }^{8,33,37,76}$ T. Sato, ${ }^{76}$ S. Sawada, ${ }^{37}$

B. K. Schmoll, ${ }^{74}$ K. Sedgwick, ${ }^{9}$ J. Seele, ${ }^{14}$ R. Seidl, ${ }^{29,64,65}$ A. Yu. Semenov, ${ }^{32}$ V. Semenov, ${ }^{28,30}$ A. Sen, ${ }^{24,32}$ R. Seto, ${ }^{9}$ D. Sharma, ${ }^{71,79}$ T. K. Shea, ${ }^{8}$ I. Shein, ${ }^{28}$ A. Shevel, ${ }^{63,70}$ T.-A. Shibata,${ }^{64,75}$ K. Shigaki, ${ }^{26}$ M. Shimomura, ${ }^{32,55,76}$ T. Shohjoh, ${ }^{76}$ K. Shoji, ${ }^{40,64}$ P. Shukla, ${ }^{5}$ A. Sickles, ${ }^{8,29,71}$ C. L. Silva, ${ }^{32,44,68}$ D. Silvermyr, ${ }^{46,60}$ C. Silvestre, ${ }^{17}$ K. S. Sim, ${ }^{38}$ B. K. Singh, ${ }^{4}$ C. P. Singh, ${ }^{4}$ V. Singh, ${ }^{4}$ M. J. Skoby, ${ }^{50}$ S. Skutnik, ${ }^{32}$ M. Slunečka, ${ }^{10,34}$ W. C. Smith, ${ }^{1}$ A. Soldatov, ${ }^{28}$ R. A. Soltz ${ }^{43}$ W. E. Sondheim, ${ }^{44}$ S. P. Sorensen ${ }^{74}$ I. V. Sourikova, ${ }^{8}$ F. Staley, ${ }^{17}$ P. W. Stankus, ${ }^{60}$ E. Stenlund, ${ }^{46}$ M. Stepanov, ${ }^{49,58, *}$ A. Ster, ${ }^{80}$ S. P. Stoll, ${ }^{8}$ T. Sugitate, ${ }^{26}$ C. Suire, ${ }^{61}$ A. Sukhanov, ${ }^{8}$ J. P. Sullivan, ${ }^{44}$ J. Sun, ${ }^{71}$ Z. Sun, ${ }^{18}$ J. Sziklai,${ }^{80}$ T. Tabaru ${ }^{65}$ S. Takagi, ${ }^{76}$ E. M. Takagui, ${ }^{68}$ A. Takahara, ${ }^{13}$ A. Taketani ${ }^{64,65}$ R. Tanabe ${ }^{76}$ K. H. Tanaka, ${ }^{37}$ Y. Tanaka, ${ }^{54}$ S. Taneja ${ }^{71}$ K. Tanida, ${ }^{33,40,64,65,69}$ M. J. Tannenbaum, ${ }^{8}$ S. Tarafdar, ${ }^{477}$ A. Taranenko, ${ }^{56,70}$ P. Tarján, ${ }^{18}$ E. Tennant, ${ }^{58}$ H. Themann, ${ }^{71}$ D. Thomas, ${ }^{1}$ T. L. Thomas, ${ }^{57}$ R. Tieulent, ${ }^{47}$ T. Todoroki ${ }^{64,65,76}$ M. Togawa, ${ }^{40,64,65}$ A. Toia, ${ }^{71}$ J. Tojo, ${ }^{64}$ L. Tomášek,${ }^{31}$ M. Tomášek, ${ }^{16,31}$ Y. Tomita, ${ }^{76}$ H. Torii, ${ }^{26,64}$ R. S. Towell, ${ }^{1}$ V-N. Tram, ${ }^{41}$ I. Tserruya ${ }^{79}$ Y. Tsuchimoto, ${ }^{13,26,64}{ }^{\text {T. Tsuji, }}{ }^{13}$ S. K. Tuli,,${ }^{4,}$ H. Tydesjö,${ }^{46}$ N. Tyurin,${ }^{28}$ Y. Ueda, ${ }^{26}$ B. Ujvari, ${ }^{18}$ C. Vale ${ }^{8,32}$ H. Valle, ${ }^{77}$ H. W. van Hecke, ${ }^{44}$ M. Vargyas,,${ }^{19,80}$ E. Vazquez-Zambrano, ${ }^{15}$ A. Veicht, ${ }^{15,29}$ J. Velkovska ${ }^{77}$ R. Vértesi, ${ }^{18,80}$ A. A. Vinogradov,${ }^{39}$ M. Virius, ${ }^{16}$ A. Vossen, ${ }^{29}$ V. Vrba, ${ }^{16,31}$ E. Vznuzdaev, ${ }^{63}$ M. Wagner, ${ }^{40,64}$ D. Walker, ${ }^{71}$ X. R. Wang,${ }^{58,65}$ D. Watanabe, ${ }^{26}$ K. Watanabe, ${ }^{76}$

Y. Watanabe, ${ }^{64,65}$ Y. S. Watanabe, ${ }^{13}$ F. Wei,${ }^{32,58}$ R. Wei, ${ }^{70}$ J. Wessels, ${ }^{51}$ S. N. White, ${ }^{8}$ N. Willis, ${ }^{61}$ D. Winter, ${ }^{15}$ S. Wolin, ${ }^{29}$ C. P. Wong, ${ }^{24}$ C. L. Woody, ${ }^{8}$ R. M. Wright, ${ }^{1}$ M. Wysocki, ${ }^{14,60}$ B. Xia, ${ }^{59}$ W. Xie,,${ }^{9,65}$ C. Xu,${ }^{58}$ Q. Xu, ${ }^{77}$

Y. L. Yamaguchi, ${ }^{13,64,65,71,78}$ K. Yamaura, ${ }^{26}$ R. Yang, ${ }^{29}$ A. Yanovich, ${ }^{28}$ Z. Yasin, ${ }^{9}$ J. Ying, ${ }^{24}$ S. Yokkaichi, ${ }^{64,65}$ J. H. Yoo, ${ }^{38}$ Z. You, ${ }^{44,62}$ G. R. Young, ${ }^{60}$ I. Younus, ${ }^{42,57}$ H. Yu, ${ }^{58}$ I. E. Yushmanov, ${ }^{39}$ W. A. Zajc, ${ }^{15}$ O. Zaudtke, ${ }^{51}$ A. Zelenski, ${ }^{7}$ C. Zhang, ${ }^{15,60}$ S. Zharko, ${ }^{67}$ S. Zhou, ${ }^{12}$ J. Zimamyi, ${ }^{80, *}$ L. Zolin, ${ }^{34}$ and L. Zou ${ }^{9}$

(PHENIX Collaboration)

\author{
${ }^{1}$ Abilene Christian University, Abilene, Texas 79699, USA \\ ${ }^{2}$ Institute of Physics, Academia Sinica, Taipei 11529, Taiwan \\ ${ }^{3}$ Department of Physics, Augustana University, Sioux Falls, South Dakota 57197, USA \\ ${ }^{4}$ Department of Physics, Banaras Hindu University, Varanasi 221005, India \\ ${ }^{5}$ Bhabha Atomic Research Centre, Bombay 400 085, India \\ ${ }^{6}$ Baruch College, City University of New York, New York, New York 10010, USA \\ ${ }^{7}$ Collider-Accelerator Department, Brookhaven National Laboratory, Upton, New York 11973-5000, USA \\ ${ }^{8}$ Physics Department, Brookhaven National Laboratory, Upton, New York 11973-5000, USA \\ ${ }^{9}$ University of California-Riverside, Riverside, California 92521, USA \\ ${ }^{10}$ Charles University, Ovocný trh 5, Praha 1, 11636 Prague, Czech Republic \\ ${ }^{11}$ Chonbuk National University, Jeonju 561-756, Korea
}


${ }^{12}$ Science and Technology on Nuclear Data Laboratory, China Institute of Atomic Energy, Beijing 102413, People's Republic of China

${ }^{13}$ Center for Nuclear Study, Graduate School of Science, University of Tokyo, 7-3-1 Hongo, Bunkyo, Tokyo 113-0033, Japan

${ }^{14}$ University of Colorado, Boulder, Colorado 80309, USA

${ }^{15}$ Columbia University, New York, New York 10027, USA and Nevis Laboratories, Irvington, New York 10533, USA

${ }^{16}$ Czech Technical University, Zikova 4, 16636 Prague 6, Czech Republic

${ }^{17}$ Dapnia, CEA Saclay, F-91191 Gif-sur-Yvette, France

${ }^{18}$ Debrecen University, H-4010 Debrecen, Egyetem tér 1, Hungary

${ }^{19}$ ELTE, Eötvös Loránd University, H-1117 Budapest, Pázmány P. s. 1/A, Hungary

${ }^{20}$ Eszterházy Károly University, Károly Róbert Campus, H-3200 Gyöngyös, Mátrai út 36, Hungary

${ }^{21}$ Ewha Womans University, Seoul 120-750, Korea

${ }^{22}$ Florida Institute of Technology, Melbourne, Florida 32901, USA

${ }^{23}$ Florida State University, Tallahassee, Florida 32306, USA

${ }^{24}$ Georgia State University, Atlanta, Georgia 30303, USA

${ }^{25}$ Hanyang University, Seoul 133-792, Korea

${ }^{26}$ Hiroshima University, Kagamiyama, Higashi-Hiroshima 739-8526, Japan

${ }^{27}$ Department of Physics and Astronomy, Howard University, Washington, DC 20059, USA

${ }^{28}$ IHEP Protvino, State Research Center of Russian Federation, Institute for High Energy Physics, Protvino 142281, Russia

${ }^{29}$ University of Illinois at Urbana-Champaign, Urbana, Illinois 61801, USA

${ }^{30}$ Institute for Nuclear Research of the Russian Academy of Sciences, prospekt 60-letiya Oktyabrya 7a, Moscow 117312, Russia

${ }^{31}$ Institute of Physics, Academy of Sciences of the Czech Republic, Na Slovance 2, 18221 Prague 8, Czech Republic

${ }^{32}$ Iowa State University, Ames, Iowa 50011, USA

${ }^{33}$ Advanced Science Research Center, Japan Atomic Energy Agency, 2-4 Shirakata Shirane,

Tokai-mura, Naka-gun, Ibaraki-ken 319-1195, Japan

${ }^{34}$ Joint Institute for Nuclear Research, Dubna 141980, Moscow Region, Russia

${ }^{35}$ Helsinki Institute of Physics and University of Jyväskylä, P.O.Box 35, FI-40014 Jyväskylä, Finland

${ }^{36}$ KAERI, Cyclotron Application Laboratory, Seoul 34057, Korea

${ }^{37}$ KEK, High Energy Accelerator Research Organization, Tsukuba, Ibaraki 305-0801, Japan

${ }^{38}$ Korea University, Seoul 02841, Korea

${ }^{39}$ National Research Center "Kurchatov Institute”, Moscow 123098, Russia

${ }^{40}$ Kyoto University, Kyoto 606-8502, Japan

${ }^{41}$ Laboratoire Leprince-Ringuet, Ecole Polytechnique, CNRS-IN2P3, Route de Saclay, F-91128 Palaiseau, France

${ }^{42}$ Physics Department, Lahore University of Management Sciences, Lahore 54792, Pakistan

${ }^{43}$ Lawrence Livermore National Laboratory, Livermore, California 94550, USA

${ }^{44}$ Los Alamos National Laboratory, Los Alamos, New Mexico 87545, USA

${ }^{45}$ LPC, Université Blaise Pascal, CNRS-IN2P3, Clermont-Fd, 63177 Aubiere Cedex, France

${ }^{46}$ Department of Physics, Lund University, Box 118, SE-221 00 Lund, Sweden

${ }^{47}$ IPNL, CNRS/IN2P3, Univ Lyon, Universit Lyon 1, F-69622 Villeurbanne, France

${ }^{48}$ University of Maryland, College Park, Maryland 20742, USA

${ }^{49}$ Department of Physics, University of Massachusetts, Amherst, Massachusetts 01003-9337, USA

${ }^{50}$ Department of Physics, University of Michigan, Ann Arbor, Michigan 48109-1040, USA

${ }^{51}$ Institut für Kernphysik, University of Münster, D-48149 Münster, Germany

${ }^{52}$ Muhlenberg College, Allentown, Pennsylvania 18104-5586, USA

${ }^{53}$ Myongji University, Yongin, Kyonggido 449-728, Korea

${ }^{54}$ Nagasaki Institute of Applied Science, Nagasaki-shi, Nagasaki 851-0193, Japan

${ }^{55}$ Nara Women's University, Kita-uoya Nishi-machi, Nara 630-8506, Japan

${ }^{56}$ National Research Nuclear University, MEPhI, Moscow Engineering Physics Institute, Moscow 115409, Russia

${ }^{57}$ University of New Mexico, Albuquerque, New Mexico 87131, USA

${ }^{58}$ New Mexico State University, Las Cruces, New Mexico 88003, USA

${ }^{59}$ Department of Physics and Astronomy, Ohio University, Athens, Ohio 45701, USA

${ }^{60}$ Oak Ridge National Laboratory, Oak Ridge, Tennessee 37831, USA

${ }^{61}$ IPN-Orsay, Université Paris-Sud, CNRS/IN2P3, Université Paris-Saclay, BP1, F-91406 Orsay, France

${ }^{62}$ Peking University, Beijing 100871, People's Republic of China

${ }^{63}$ PNPI, Petersburg Nuclear Physics Institute, Gatchina 188300, Leningrad region, Russia

${ }^{64}$ RIKEN Nishina Center for Accelerator-Based Science, Wako, Saitama 351-0198, Japan

${ }^{65}$ RIKEN BNL Research Center, Brookhaven National Laboratory, Upton, New York 11973-5000, USA

${ }^{66}$ Physics Department, Rikkyo University, 3-34-1 Nishi-Ikebukuro, Toshima, Tokyo 171-8501, Japan

${ }^{67}$ Saint Petersburg State Polytechnic University, St. Petersburg 195251, Russia

${ }^{68}$ Universidade de São Paulo, Instituto de Física, Caixa Postal 66318, São Paulo CEP05315-970, Brazil

${ }^{69}$ Department of Physics and Astronomy, Seoul National University, Seoul 151-742, Korea

${ }^{70}$ Chemistry Department, Stony Brook University, SUNY, Stony Brook, New York 11794-3400, USA 


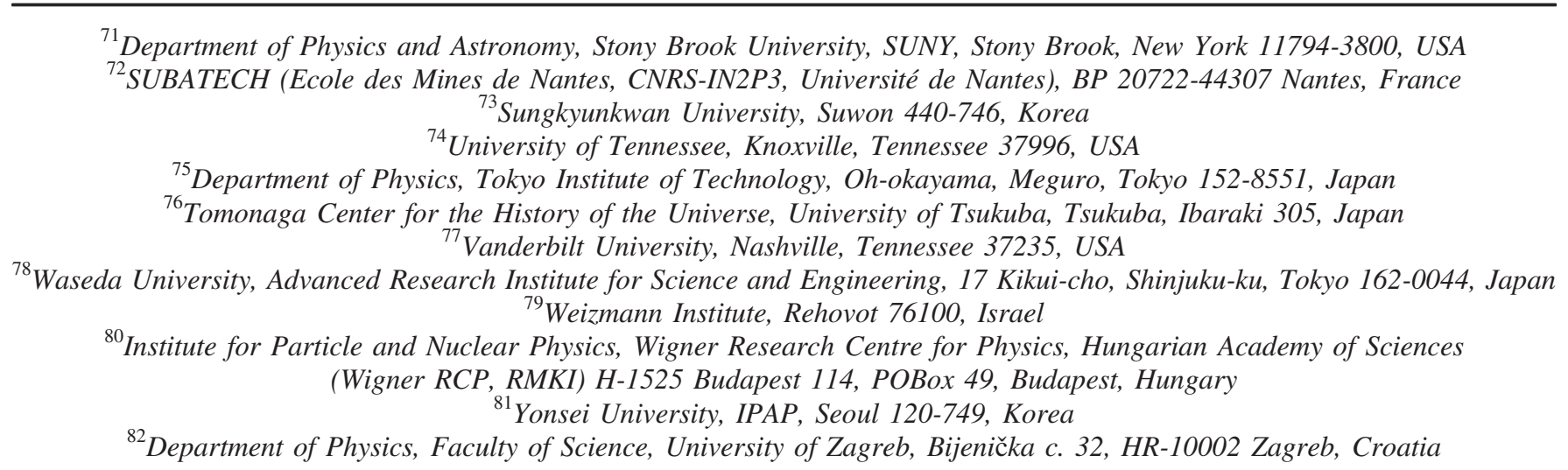

(Received 15 May 2018; revised manuscript received 27 April 2019; published 10 July 2019)

The PHENIX collaboration presents first measurements of low-momentum $\left(0.4<p_{T}<3 \mathrm{GeV} / c\right)$ direct-photon yields from $\mathrm{Au}+\mathrm{Au}$ collisions at $\sqrt{s_{N N}}=39$ and $62.4 \mathrm{GeV}$. For both beam energies the direct-photon yields are substantially enhanced with respect to expectations from prompt processes, similar to the yields observed in $\mathrm{Au}+\mathrm{Au}$ collisions at $\sqrt{s_{N N}}=200$. Analyzing the photon yield as a function of the experimental observable $d N_{\mathrm{ch}} / d \eta$ reveals that the low-momentum $(>1 \mathrm{GeV} / c)$ direct-photon yield $d N_{\gamma}^{\text {dir }} / d \eta$ is a smooth function of $d N_{\mathrm{ch}} / d \eta$ and can be well described as proportional to $\left(d N_{\mathrm{ch}} / d \eta\right)^{\alpha}$ with $\alpha \approx 1.25$. This scaling behavior holds for a wide range of beam energies at the Relativistic Heavy Ion Collider and the Large Hadron Collider, for centrality selected samples, as well as for different $A+A$ collision systems. At a given beam energy, the scaling also holds for high $p_{T}(>5 \mathrm{GeV} / c)$, but when results from different collision energies are compared, an additional $\sqrt{s_{N N}}$-dependent multiplicative factor is needed to describe the integrated-direct-photon yield.

DOI: 10.1103/PhysRevLett.123.022301

Measurements of direct photons provide information about the strongly coupled quark-gluon plasma (QGP) produced in heavy ion collisions and its "fireball" evolution to hadron resonance matter. Owing to their long mean-free path, photons do not interact with the matter, and thus, their spectra provide information about all stages of the collision integrated over space and time [1-3]. In particular, low $p_{T}$ photons in the momentum range up to a few $\mathrm{GeV} / c$ are expected to carry information about the hot and dense fireball.

In experiments, direct photons are detected simultaneously with a much larger number of photons from hadron decays, mostly from $\pi^{0}$ and $\eta$ mesons. The main challenge is to subtract these decay contributions from the measurement to obtain the photons directly emitted from the collision. In addition to photons from the hot fireball, direct photons include those emitted from initial hard scattering processes, such as quark-gluon Compton scattering among the incoming partons [4]. Disentangling this prompt component from the photons emitted from the fireball is an additional challenge.

Published by the American Physical Society under the terms of the Creative Commons Attribution 4.0 International license. Further distribution of this work must maintain attribution to the author(s) and the published article's title, journal citation, and DOI. Funded by SCOAP ${ }^{3}$.
First evidence for direct photon emission from heavy ion collisions came from WA98 [5,6], with conclusive results only for $p_{T}>1.5 \mathrm{GeV} / c$. PHENIX established that a large number of low $p_{T}$ direct photons are radiated from the fireball created in $\mathrm{Au}+\mathrm{Au}$ collisions at $\sqrt{s_{N N}}=200 \mathrm{GeV}$ [7] and that their yield increases with a power of $N_{\text {part }}$ while the inverse slopes of the spectra are independent of the centrality of the collisions [8]. Simultaneously, low $p_{T}$ direct photon emission exhibits a significant azimuthal anisotropy with respect to the reaction plane $[9,10]$.

ALICE has published [11,12] similar observations of low $p_{T}$ : direct photons from $\mathrm{Pb}+\mathrm{Pb}$ collisions at $\sqrt{s_{N N}}=2760 \mathrm{GeV}$. STAR also reported a measurement of the direct photon yields in $\mathrm{Au}+\mathrm{Au}$ at $\sqrt{s_{N N}}=$ $200 \mathrm{GeV}$ [13], the published yields are significantly lower compared to PHENIX results. The origin of the discrepancy remains unresolved $[14,15]$.

A large body of theoretical work on low $p_{T}$ direct photon emission in $\mathrm{A}+\mathrm{A}$ collisions exists in the literature. Many model calculations are qualitatively consistent with the data, but a quantitative description remains difficult, primarily due to the simultaneous observation of large yields and large azimuthal anisotropies [16-39].

To provide further insights, PHENIX is investigating the system size dependence of direct photon emission from heavy ion collisions by varying beam energy, centrality, and collision species. In this Letter, we present low- $p_{T}$ 
direct photon data from $\mathrm{Au}+\mathrm{Au}$ collisions at $\sqrt{s_{N N}}=39$ and $62.4 \mathrm{GeV}$ taken with the PHENIX experiment in 2010. We compare the centrality selected spectra and integrated yields from $\mathrm{Au}+\mathrm{Au}$ to those from $p+p$ collisions at $\sqrt{s_{N N}}=200 \mathrm{GeV}[7,8], \mathrm{Cu}+\mathrm{Cu}$ collisions at $\sqrt{s_{N N}}=$ $200 \mathrm{GeV}$ [40], and $\mathrm{Pb}+\mathrm{Pb}$ collisions at $\sqrt{s_{N N}}=$ $2760 \mathrm{GeV}$ [11]. This study covers a factor of 70 in $\sqrt{s_{N N}}$ and nearly 2 orders of magnitude in system size.

The 39 and $62.4 \mathrm{GeV}$ direct photon spectra are obtained from two data samples of minimum bias (MB) $\mathrm{Au}+\mathrm{Au}$ collisions that have a total of $7.79 \times 10^{7}$ and $2.12 \times 10^{8}$ events, respectively. The MB trigger and centrality selection is derived from data taken with the PHENIX beambeam counters [41]. The data analysis uses the same techniques deployed for the analysis of the $\sqrt{s_{N N}}=$ $200 \mathrm{GeV} \mathrm{Au}+\mathrm{Au}$ data [8], which were taken in the same year under nearly identical conditions. Here, we give a brief overview of the setup and data analysis, and refer to our previous publication for more details [8].

Photons are reconstructed through their conversion to $e^{+} e^{-}$pairs in the detector material, specifically the readout boards of the hadron blind detector (HBD) [42] that are located at a radius of $60 \mathrm{~cm}$ from the beam axes. The trajectories and momenta of the $e^{+}$and $e^{-}$are determined by the central arm tracking detectors [43]. Each of the two central arms covers $90^{\circ}$ in azimuth and a rapidity range of $|\eta|<0.35$. A transverse momentum cut, $p_{T}>200 \mathrm{MeV} / c$, is applied to each trajectory. To identify trajectories as $e^{+}$or $e^{-}$candidates, we require a minimum of three associated signals in the ring-imaging Čerenkov detector [44] and that the energy measured in the electromagnetic calorimeter (EMCal) [45] matches the measured momentum $(E / p>0.5)$.

All $e^{+}$and $e^{-}$reconstructed in the same arm are matched to pairs. In the 2010 setup, there is no tracking near the collision point, so the origin of an individual track is unknown. Thus, for each $e^{+} e^{-}$pair, the mass is calculated twice: first, assuming the pair originated at the event vertex $\left(m_{\mathrm{vtx}}\right)$, then assuming the $e^{+} e^{-}$is a conversion pair from the HBD readout boards $\left(m_{\mathrm{HBD}}\right)$. In the latter case, $m_{\mathrm{HBD}}$ will be consistent with zero, within a mass resolution of a few $\mathrm{MeV} / c^{2}$, while $m_{\mathrm{vtx}}$ will be about $12 \mathrm{MeV} / c^{2}$. With a cut on both masses a sample of photon conversion is selected with a purity of about $99 \%$. The combinatorial background is negligible, because the conversion material, in radiation length $X / X_{0} \approx 3 \%$, is about 10 times thicker than materials closer to the vertex, and it is at a relatively large distance from the event vertex. The $1 \%$ contamination is mostly from $\pi^{0}$ Dalitz decays, $\pi^{0} \rightarrow \gamma e^{+} e^{-}$, and from conversions in front of the HBD readout boards.

The direct photon content in the photon sample is determined by the ratio $R_{\gamma}$, which is the ratio of all emitted photons $\left(\gamma^{\text {incl }}\right)$ to those from hadron decays $\left(\gamma^{\text {hadron }}\right)$. The ratio $R_{\gamma}$ is determined from a double ratio

$$
R_{\gamma}=\frac{\gamma^{\text {incl }}}{\gamma^{\text {hadron }}}=\frac{\left\langle\varepsilon_{\gamma} f\right\rangle\left(N_{\gamma}^{\text {incl }} / N_{\gamma}^{\pi^{0} \text {,tag }}\right)_{\text {Data }}}{\left(\gamma^{\text {hadron }} / \gamma^{\pi^{0}}\right)_{\text {Sim }}} .
$$

All quantities in this double ratio are functions of the conversion photon $p_{T}^{e e}$. The measured quantities are the number of detected conversion photons $N_{\gamma}^{\text {incl }}$ and the subset of those that are tagged as $\pi^{0}$ decay photon $N_{\gamma}^{\pi^{0}}$,tag . The tagged photons $N_{\gamma}^{\pi^{0}}$,tag are determined statistically in bins of the $p_{T}^{e e}$. Each conversion photon is paired with all showers with $E>400 \mathrm{MeV}$ measured in the EMCal of the same arm. The invariant $e^{+} e^{-} \gamma$ mass is calculated and the counts above the combinatorial background in the $\pi^{0}$ mass peak give $N_{\gamma}^{\pi^{0}, \text { tag }}$. To convert the ratio $N_{\gamma}^{\text {incl }} / N_{\gamma}^{\pi^{0}, \text { tag }}$ to $\gamma^{\text {incl }} / \gamma^{\pi^{0}}$ only $N_{\gamma}^{\pi^{0}, \text { tag }}$ needs to be corrected for the momentum averaged conditional acceptance efficiency $\left\langle\varepsilon_{\gamma} f\right\rangle$ that the second decay photon can be reconstructed in the EMCal. All other corrections to the numerator and denominator cancel [8]. Because rather loose cuts are applied to the EMCal showers, $\left\langle\varepsilon_{\gamma} f\right\rangle$ is mostly determined by the $\pi^{0}$ decay kinematics, the detector geometry, and the energy cut. Thus, $\left\langle\varepsilon_{\gamma} f\right\rangle$ can be calculated to a few percent accuracy using a Monte Carlo simulation of $\pi^{0}$ decays. Photons from pions are determined from the measured $\pi^{0}$ spectra [46] and two body decay kinematics. The spectrum of decay photons $\left(\gamma^{\text {hadron }}\right)$ is derived from $\gamma^{\pi^{0}}$ and the $\eta / \pi^{0}$ ratio [47], which is independent of collision system and energy, with additional contribution from heavier mesons of about $4 \%$.

Once $R_{\gamma}$ is established, the direct photon spectrum can be calculated as

$$
\gamma^{\text {direct }}=\left(R_{\gamma}-1\right) \gamma^{\text {hadron }} .
$$

The uncertainty on $\gamma^{\text {hadron }}$ approximately $10 \%$ [8], cancels in $R_{\gamma}$ [with that of $\gamma^{\pi^{0}}$ in Eq. (1)] but has to be applied to $\gamma^{\text {direct. }}$. The systematic uncertainties on the 39 and $62.4 \mathrm{GeV}$ data are similar in magnitude to those for $200 \mathrm{GeV}$ presented in [8]. For integrated yield, we treat every systematic uncertainty as $p_{T}$-correlated in the interest of consistency throughout the different data sets.

Figure 1 shows the invariant yield of direct photons normalized to $\left(d N_{\mathrm{ch}} / d \eta\right)^{1.25}$, this normalization is discussed below. Panel (a) shows $\mathrm{Au}+\mathrm{Au} \mathrm{MB}$ data at $\sqrt{s_{N N}}=62.4$ and $39 \mathrm{GeV}$, panel (b) gives $\mathrm{Au}+\mathrm{Au}$ data in three centrality classes at $200 \mathrm{GeV}$, and panel (c) compares data from different beam energies and systems. Below $3 \mathrm{GeV} / c$ the 62.4 and $39 \mathrm{GeV}$ data show substantial direct photon yields, which are comparable in magnitude and spectral shape, albeit within large uncertainties. For $62.4 \mathrm{GeV}$, we can also extract a direct photon signal for $0 \%-20 \%$ and $20 \%-40 \%$ centrality selection and find that the direct photon yield increases with centrality. 


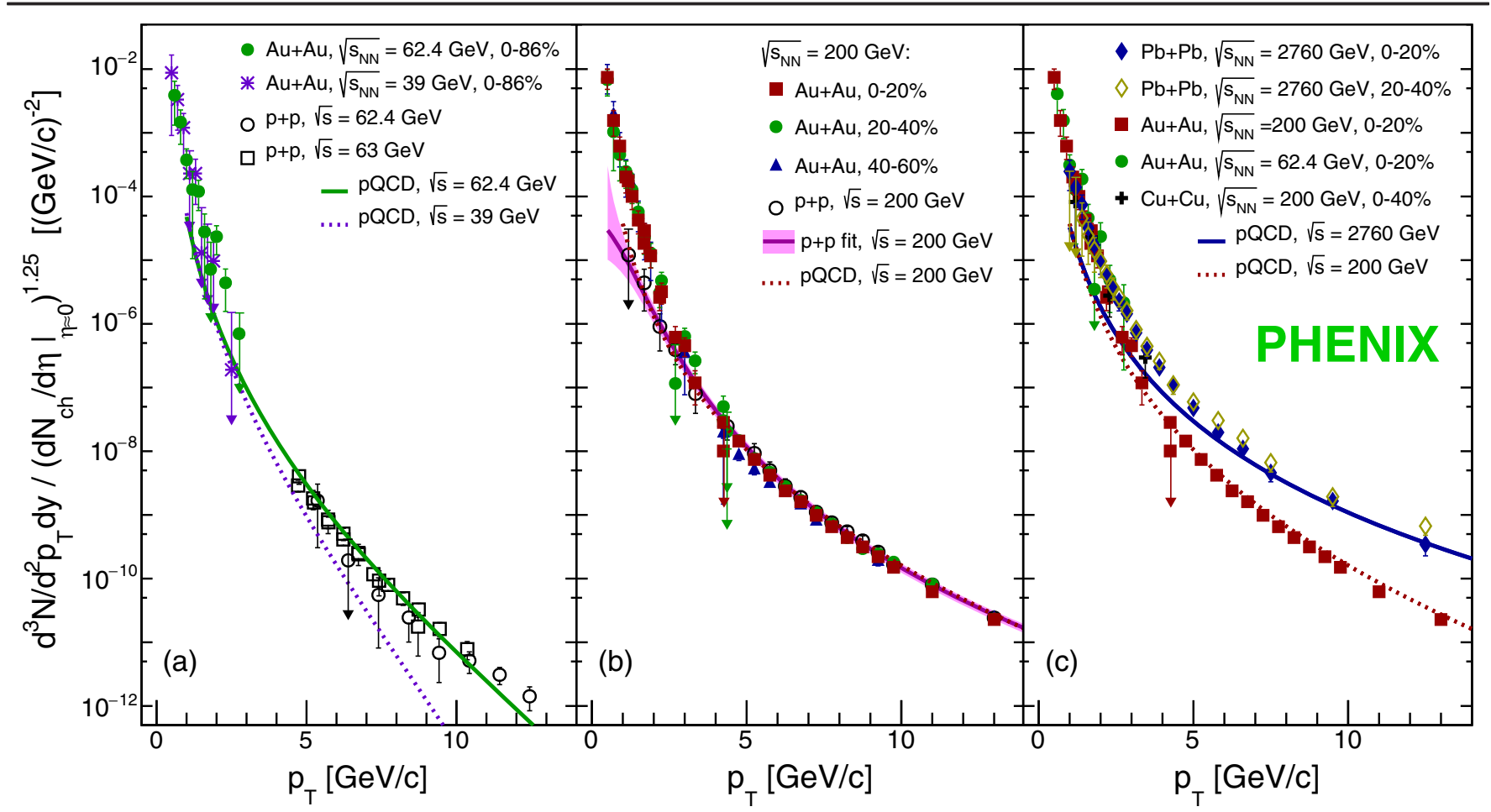

FIG. 1. Direct photon spectra normalized by $\left(d N_{\mathrm{ch}} / d \eta\right)^{1.25}$ for $\mathrm{Au}+\mathrm{Au}$ at 39 and $64.2 \mathrm{GeV}$ (a) and (b) at $200 \mathrm{GeV}$ [8]; panel (c) compares for different $A+A$ systems at different $\sqrt{s_{N N}}[11,40]$. Panels (a) and (b) also show $p+p$ data [8,48-50]. All panels show pQCD calculations for the corresponding $\sqrt{s}$ [21,51]. The errors shown are the quadratic sum of systematic and statistical uncertainties. Uncertainties on the $d N_{\mathrm{ch}} / d \eta$ are not included.

All observations are similar to those already published for $\mathrm{Au}+\mathrm{Au}$ collisions at $\sqrt{s_{N N}}=200 \mathrm{GeV}$ [8].

To compare data from different beam energies, collisions species, and collision centralities, we use the measured charged particle multiplicity $d N_{\mathrm{ch}} / d \eta$ as a measure of the system size at hadronization. For a fixed beam energy $d N_{\mathrm{ch}} / d \eta$ is roughly proportional $N_{\text {part }}$. However, unlike $N_{\text {part }}, d N_{\text {ch }} / d \eta$ does not saturate but increases monotonically with beam energy for collisions of the same nuclei at the same impact parameter.

Direct photon production at high $p_{T}$ results from hard scattering, which, at a fixed $\sqrt{s_{N N}}$, scales with the number of binary collisions $N_{\text {coll }}$. We find that $N_{\text {coll }}$ exhibits a remarkably simple relation with the $d N_{\mathrm{ch}} / d \eta$ that takes the form

$$
N_{\text {coll }}=\frac{1}{\mathrm{SY}\left(\sqrt{s_{N N}}\right)} \times\left(\frac{d N_{\mathrm{ch}}}{d \eta}\right)^{\alpha} .
$$

This is shown in Fig. 2 where $N_{\text {coll }}$ is plotted vs $d N_{\mathrm{ch}} / d \eta$ for different $\sqrt{s_{N N}}$. PHENIX data are taken from [52] and ALICE data at $\sqrt{s_{N N}}=2760 \mathrm{GeV}$ are from [53]. The exponent $\alpha$ is determined through a simultaneous fit to all data shown in Fig. 2 and found to be $\alpha=1.25 \pm 0.02$. The specific yield SY increases logarithmically with $\sqrt{s_{N N}}$ as $\mathrm{SY}\left(\sqrt{s_{N N}}\right)=(0.976 \pm 0.054) \log \left(\sqrt{s_{N N}}\right)-(1.827 \pm 0.253)$.

Figure 1 depicts the direct photon yield for different beam energies and centralities normalized by $\left(d N_{\mathrm{ch}} / d \eta\right)^{1.25}$. In panel (b), three different centrality selections of $\mathrm{Au}+\mathrm{Au}$ collisions at $\sqrt{s_{N N}}=200 \mathrm{GeV}$ are shown together with data from $p+p$ at the same beam energy. The normalized spectra from $\mathrm{Au}+\mathrm{Au}$ are very similar for all three centrality

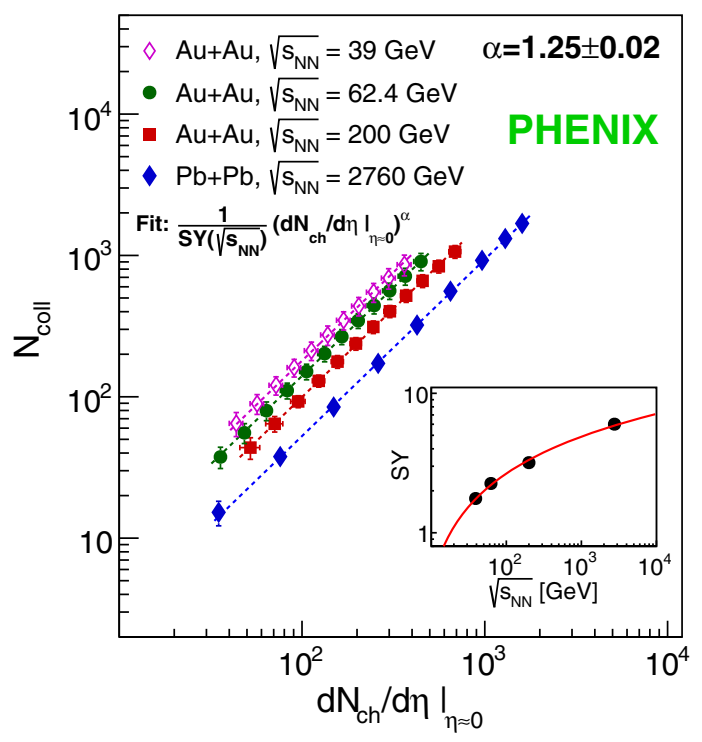

FIG. 2. Number of binary collisions, $N_{\text {coll }}$ vs $d N_{\mathrm{ch}} / d \eta$, for four beam energies. The errors shown reflect the uncertainty of $N_{\text {coll }}$ from the Glauber calculation. Fitting Eq. (3) simultaneously to all data with a common $\alpha$ results in $\alpha=1.25$ and a $\sqrt{s_{N N}}$ dependence SY as shown in the text below Eq. (3). 
selections. Above $3-4 \mathrm{GeV} / c$, the normalized yield is the same as for $p+p$ collisions and can be reproduced by perturbative quantum chromodynamics (pQCD) calculations with a renormalization and factorization scale of $\mu=$ $0.5 p_{T}[51,54]$. Here, the pQCD calculation was normalized to the experimental $d N_{\mathrm{ch}} / d \eta$ for $\sqrt{s}=200 \mathrm{GeV}$ from [55]. Also shown in Fig. 1(b) is an empirical fit to the $p+p$ data [56] of the form $a\left(1+p_{T}^{2} / b\right)^{c}$ [40]. Below $2-3 \mathrm{GeV} / c$, the normalized yield in $\mathrm{Au}+\mathrm{Au}$ collisions is significantly enhanced compared to that in $p+p$ collisions, but follows the same scaling behavior with $\left(d N_{\mathrm{ch}} / d \eta\right)^{1.25}$ independent of centrality.

Panels (a) and (c) of Fig. 1 show that for $p_{T}$ below $2-3 \mathrm{GeV} / c$ the same scaling with $\left(d N_{\mathrm{ch}} / d \eta\right)^{1.25}$ occurs for different $\sqrt{s_{N N}}$ and collisions systems. Below $2 \mathrm{GeV} / c$ the spectra have very similar shapes. We note that the apparent difference of the inverse slopes reported by PHENIX [8] and ALICE [11] is largely due to the different fit ranges used [57].

At higher $p_{T}$, the expected difference with $\sqrt{s_{N N}}$ is observed. As for $\sqrt{s_{N N}}=200 \mathrm{GeV}$, at high $p_{T}$, the $2760 \mathrm{GeV}$ data are well reproduced by the pQCD calculation, though only above $5-6 \mathrm{GeV} / c$ rather than 3-4 GeV/c. Note that the extrapolated $\mathrm{pQCD}$ calculations for $p+p$ at different $\sqrt{s}$ seem to converge to the same normalized yield at low $p_{T}$, but at a tenth of the $A+A$ yield.

We quantify direct photon emission by integrating the invariant yield above $p_{T}=1.0 \mathrm{GeV} / c$ and $p_{T}=5.0 \mathrm{GeV} / c$. The integrals with the lower threshold will be dominated by excess low $p_{T}$ photons unique to $A+A$ collisions, while the integrals with the higher threshold are more sensitive to photons from initial hard scattering processes. The results are shown in Figs. 3 and 4 as a function of $d N_{\mathrm{ch}} / d \eta$. Also plotted are power-law functions $A\left(d N_{\mathrm{ch}} / d \eta\right)^{\alpha}$ with fixed $\alpha=1.25$ and a normalization fitted to the data.

For $A+A$ collisions, the integrated yields for the $1.0 \mathrm{GeV} / c$ threshold, shown in Fig. 3, scale as (7.140土 $0.265) \times 10^{-4} \times\left(d N_{\mathrm{ch}} / d \eta\right)^{1.250}$. We find the same scaling if $\alpha$ is not constrained: $(8.300 \pm 1.680) \times 10^{-4} \times$ $\left(d N_{\mathrm{ch}} / d \eta\right)^{1.225 \pm 0.034}$. The $A+A$ points are compared to the integrated yield for $\sqrt{s}=200 \mathrm{GeV} p+p$ obtained from the fit to the data, which is scaled with $N_{\text {coll }}$ to the corresponding $d N_{\mathrm{ch}} / d \eta$ for each $\sqrt{s_{N N}}=200 \mathrm{GeV} A+A$ point. The width of the band is given by the combined uncertainties on the fit function and $N_{\text {coll }}$. It is parallel to the $A+A$ trend but lower by about an order of magnitude. Also shown are the scaled integrated yields from pQCD calculations for $\sqrt{s}=62.4$, 200 , and $2760 \mathrm{GeV}$, consistent with the band independent of beam energy.

For the $p_{T}$ threshold of $5 \mathrm{GeV} / c$ the integrated yields from $\mathrm{Au}+\mathrm{Au}$ and $p+p$ at $200 \mathrm{GeV}$ follow the same $\left(d N_{\mathrm{ch}} / d \eta\right)^{1.25}$ trend, and are described by the pQCD calculation. The $2760 \mathrm{GeV}$ data are also consistent with $\left(d N_{\mathrm{ch}} / d \eta\right)^{1.25}$ but show a significantly higher yield than at

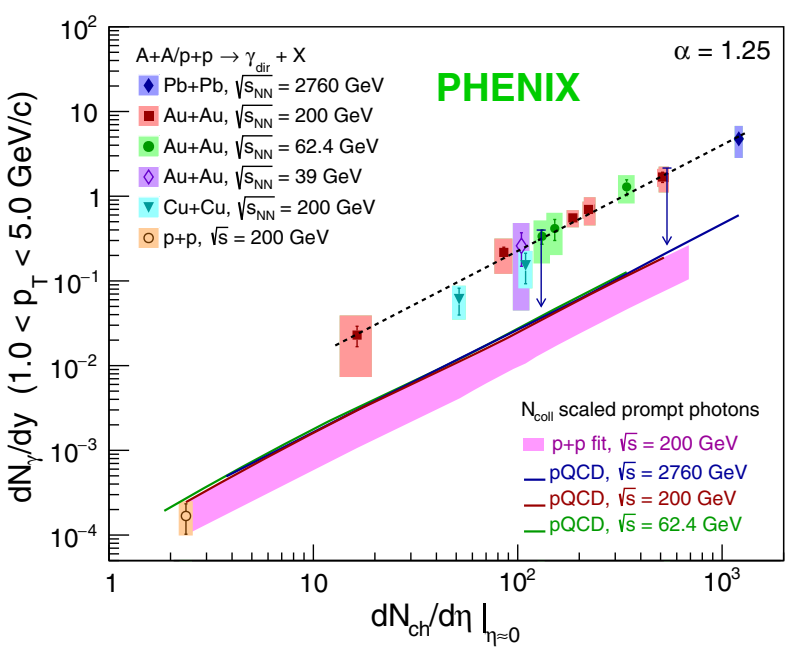

FIG. 3. Integrated direct photon yield $\left(p_{T}>1.0 \mathrm{GeV} / c\right)$ vs $d N_{\mathrm{ch}} / d \eta$, for data sets shown in Fig. 1. The dashed line is a power law fit with a fixed slope of $\alpha=1.25$. The two upper limits correspond to the data in $20 \%-40 \%$ and $40 \%-80 \% \mathrm{~Pb}+\mathrm{Pb}$ collisions at $\sqrt{s_{N N}}=2760 \mathrm{GeV}$. The integrated yields of the fit to $p+p$ data and of the $\mathrm{pQCD}$ calculations are shown as well, both scaled by $N_{\text {coll }}[21,51]$.

$200 \mathrm{GeV}$ data at the same $d N_{\mathrm{ch}} / d \eta$. The $N_{\text {coll }}$ scaled pQCD calculation is about $30 \%$ below the data, which may not be significant considering the $25 \%$ systematic uncertainty on the calculation.

While the functional form $A\left(d N_{\mathrm{ch}} / d \eta\right)^{\alpha}$ describes the integrated direct photon yields well, it is not unique. For instance, the data can be equally well fitted by $A\left(d N_{\mathrm{ch}} / d \eta\right)+B\left(d N_{\mathrm{ch}} / d \eta\right)^{4 / 3}$ [58]. For the data in Fig. 3, this fit results in parameters $A=(8.68 \pm 3.06) \times$ $10^{-4}$ and $B=(3.09 \pm 0.45) \times 10^{-4}$. The important point is

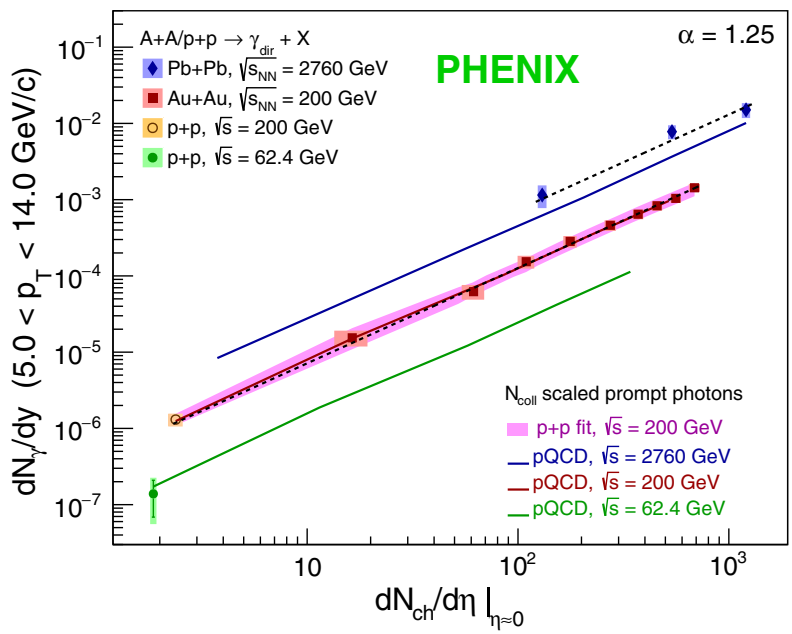

FIG. 4. Integrated direct photon yield $\left(p_{T}>5.0 \mathrm{GeV} / c\right)$ vs $d N_{\mathrm{ch}} / d \eta$, for different data sets. The dashed lines show power law fits to the data with fixed slope of $\alpha=1.25$. Integrated yields from $\mathrm{pQCD}$ calculations scaled by $N_{\text {coll }}$ are also shown. 
that $A+A$ data from different centralities and a wide range of collision energies can be empirically described in terms of $d N_{\mathrm{ch}} / d \eta$ with just two parameters, suggesting some fundamental commonality in the underlying physics.

There are two main conclusions from the analyses presented in this Letter. (i) At a given beam energy, the direct photon yield scales with $d N_{\mathrm{ch}} / d \eta^{1.25}$ or $N_{\text {coll }}$ for all observed $p_{T}$. There seems to be no qualitative change in the photon sources and/or their relative contributions for different collision centrality or system size. (ii) From $\sqrt{s_{N N}}=39$ to $2760 \mathrm{GeV}$ the same scaling is observed for $p_{T}<2 \mathrm{GeV} / c$. This suggests that the main sources contributing to this $p_{T}$ range are also very similar across beam energies.

If thermal radiation is the source of low $p_{T}$ direct photons, the similarity at the same $d N_{\mathrm{ch}} / d \eta$ across beam energies and centralities for $p_{T} \lesssim 2 \mathrm{GeV} / c$, suggests that the bulk of the matter that emits the radiation is similar in terms of temperature and space time evolution. This would be natural, if most of the photons are emitted near the transition from QGP to hadrons.

While at high $p_{T}$, the scaled yields in $p+p$ and $A+A$ are identical, at low $p_{T}$ they differ by a factor of 10 . This implies that there must be a transition from the small $p+p$ yield to the enhanced $A+A$-like low $p_{T}$ yields in the $d N_{\mathrm{ch}} / d \eta$ range of $\approx 2$ to 20 , which will be accessible with the data taken by PHENIX with small systems $p+\mathrm{Au}$, $d+\mathrm{Au}$, and ${ }^{3} \mathrm{He}+\mathrm{Au}$.

We thank the staff of the Collider-Accelerator and Physics Departments at Brookhaven National Laboratory and the staff of the other PHENIX participating institutions for their vital contributions. We acknowledge support from the Office of Nuclear Physics in the Office of Science of the Department of Energy, the National Science Foundation, Abilene Christian University Research Council, Research Foundation of SUNY, and Dean of the College of Arts and Sciences, Vanderbilt University (U.S.A), Ministry of Education, Culture, Sports, Science, and Technology and the Japan Society for the Promotion of Science (Japan), Conselho Nacional de Desenvolvimento Científico e Tecnológico and Fundação de Amparo à Pesquisa do Estado de São Paulo (Brazil), Natural Science Foundation of China (People's Republic of China), Croatian Science Foundation and Ministry of Science and Education (Croatia), Ministry of Education, Youth and Sports (Czech Republic), Centre National de la Recherche Scientifique, Commissariat à l'Énergie Atomique, and Institut National de Physique Nucléaire et de Physique des Particules (France), Bundesministerium für Bildung und Forschung, Deutscher Akademischer Austausch Dienst, and Alexander von Humboldt Stiftung (Germany), J. Bolyai Research Scholarship, EFOP, the New National Excellence Program (ÚNKP), NKFIH, and OTKA (Hungary), Department of Atomic Energy and Department of Science and Technology (India), Israel
Science Foundation (Israel), Basic Science Research Program through NRF of the Ministry of Education (Korea), Physics Department, Lahore University of Management Sciences (Pakistan), Ministry of Education and Science, Russian Academy of Sciences, Federal Agency of Atomic Energy (Russia), VR and Wallenberg Foundation (Sweden), the U.S. Civilian Research and Development Foundation for the Independent States of the Former Soviet Union, the Hungarian American Enterprise Scholarship Fund, the US-Hungarian Fulbright Foundation, and the US-Israel Binational Science Foundation.

*Deceased.

†PHENIX Spokesperson. akiba@rcf.rhic.bnl.gov

[1] P. Stankus, Direct photon production in relativistic heavyion collisions, Annu. Rev. Nucl. Part. Sci. 55, 517 (2005).

[2] G. David, R. Rapp, and Z. Xu, Electromagnetic probes at RHIC-II, Phys. Rep. 462, 176 (2008).

[3] O. Linnyk, E. L. Bratkovskaya, and W. Cassing, Effective QCD and transport description of dilepton and photon production in heavy-ion collisions and elementary processes, Prog. Part. Nucl. Phys. 87, 50 (2016).

[4] H. Fritzsch and P. Minkowski, Measuring QCD Compton effects, Phys. Lett. 69B, 316 (1977).

[5] M. M. Aggarwal et al. (WA98 Collaboration), Interferometry of Direct Photons in Central Pb-208+Pb-208 Collisions at 158-A-GeV, Phys. Rev. Lett. 93, 022301 (2004).

[6] M. M. Aggarwal et al. (WA98 Collaboration), Observation of Direct Photons in Central 158-A-GeV Pb-208+Pb-208 Collisions, Phys. Rev. Lett. 85, 3595 (2000).

[7] A. Adare et al. (PHENIX Collaboration), Enhanced Production of Direct Photons in $\mathrm{Au}+\mathrm{Au}$ Collisions at $\sqrt{s_{N N}}=$ $200 \mathrm{GeV}$ and Implications for the Initial Temperature, Phys. Rev. Lett. 104, 132301 (2010).

[8] A. Adare et al. (PHENIX Collaboration), Centrality dependence of low-momentum direct-photon production in $\mathrm{Au}+\mathrm{Au}$ collisions at $\sqrt{s_{N N}}=200 \mathrm{GeV}$, Phys. Rev. C 91, 064904 (2015).

[9] A. Adare et al. (PHENIX Collaboration), Observation of Direct-Photon Collective Flow in $\sqrt{s_{N N}}=200 \mathrm{GeV}$ $\mathrm{Au}+\mathrm{Au}$ Collisions, Phys. Rev. Lett. 109, 122302 (2012).

[10] A. Adare et al. (PHENIX Collaboration), Azimuthally anisotropic emission of low-momentum direct photons in $\mathrm{Au}+\mathrm{Au}$ collisions at $\sqrt{s_{N N}}=200 \mathrm{GeV}$, Phys. Rev. C 94, 064901 (2016).

[11] J. Adam et al. (ALICE Collaboration), Direct photon production in $\mathrm{Pb}-\mathrm{Pb}$ collisions at $\sqrt{s_{N N}}=2760 \mathrm{GeV}$, Phys. Lett. B 754, 235 (2016).

[12] D. Lohner (ALICE Collaboration), Measurement of direct-photon elliptic flow in $\mathrm{Pb}-\mathrm{Pb}$ collisions at $\sqrt{s_{N N}}=2760 \mathrm{GeV}$, J. Phys. Conf. Ser. 446, 012028 (2013).

[13] L. Adamczyk et al. (STAR Collaboration), Direct virtual photon production in $\mathrm{Au}+\mathrm{Au}$ collisions at $\sqrt{s_{N N}}=$ $200 \mathrm{GeV}$, Phys. Lett. B 770, 451 (2017).

[14] We note that PHENIX has published consistent results from several independent analyses with different methods, using 
virtual photons [7], which is the method adopted by STAR [13], and using photon conversions in the detector material [8]. A third method using photons measured through their energy deposited in the electromagnetic calorimeter to reconstruct low $p_{T}$ photons has not been published [15], but gives consistent results as well.

[15] H. Gong, Measurement of direct photons in ultra-relativistic $\mathrm{Au}+\mathrm{Au}$ collisions, Ph.D. thesis, Stony Brook University, 2014.

[16] H. van Hees, C. Gale, and R. Rapp, Thermal photons and collective flow at the relativistic heavy-ion collider, Phys. Rev. C 84, 054906 (2011).

[17] H. van Hees, Min He, and R. Rapp, Pseudo-critical enhancement of thermal photons in relativistic heavy-ion collisions?, Nucl. Phys. A933, 256 (2015).

[18] M. Dion, J.-F. Paquet, B. Schenke, C. Young, S. Jeon, and C. Gale, Viscous photons in relativistic heavy ion collisions, Phys. Rev. C 84, 064901 (2011).

[19] C. Shen, U. W. Heinz, J.-F. Paquet, and C. Gale, Thermal photons as a quark-gluon plasma thermometer reexamined, Phys. Rev. C 89, 044910 (2014).

[20] C. Shen, J. F. Paquet, G. S. Denicol, S. Jeon, and C. Gale, Thermal Photon Radiation in High Multiplicity $\mathrm{p}+\mathrm{Pb}$ Collisions at the Large Hadron Collider, Phys. Rev. Lett. 116, 072301 (2016).

[21] J. F. Paquet, C. Shen, G. S. Denicol, M. Luzum, B. Schenke, $\mathrm{S}$. Jeon, and C. Gale, Production of photons in relativistic heavy-ion collisions, Phys. Rev. C 93, 044906 (2016).

[22] E. L. Bratkovskaya, S. M. Kiselev, and G. B. Sharkov, Direct photon production from hadronic sources in highenergy heavy-ion collisions, Phys. Rev. C 78, 034905 (2008).

[23] E. L. Bratkovskaya, Phenomenology of photon and dilepton production in relativistic nuclear collisions, Nucl. Phys. A931, 194 (2014).

[24] O. Linnyk, W. Cassing, and E. L. Bratkovskaya, Centrality dependence of the direct photon yield and elliptic flow in heavy-ion collisions at $\sqrt{s_{N N}}=200 \mathrm{GeV}$, Phys. Rev. C 89, 034908 (2014).

[25] M. Chiu, T. K. Hemmick, V. Khachatryan, A. Leonidov, J. Liao, and L. McLerran, Production of photons and dileptons in the glasma, Nucl. Phys. A900, 16 (2013).

[26] L. McLerran and B. Schenke, The glasma, photons and the implications of anisotropy, Nucl. Phys. A929, 71 (2014).

[27] L. McLerran and B. Schenke, A tale of tails: Photon rates and flow in ultra-relativistic heavy ion collisions, Nucl. Phys. A946, 158 (2016).

[28] J. Berges, K. Reygers, N. Tanji, and R. Venugopalan, Parametric estimate of the relative photon yields from the glasma and the quark-gluon plasma in heavy-ion collisions, Phys. Rev. C 95, 054904 (2017).

[29] A. Monnai, Thermal photon $v_{2}$ with slow quark chemical equilibration, Phys. Rev. C 90, 021901(R) (2014).

[30] C.-H. Lee and I. Zahed, Electromagnetic radiation in hot QCD matter: Rates, electric conductivity, flavor susceptibility and diffusion, Phys. Rev. C 90, 025204 (2014).

[31] S. Turbide, R. Rapp, and C. Gale, Hadronic production of thermal photons, Phys. Rev. C 69, 014903 (2004).
[32] K. Dusling and I. Zahed, Thermal photons from heavy ion collisions: A spectral function approach, Phys. Rev. C 82, 054909 (2010).

[33] M. Heffernan, P. Hohler, and R. Rapp, Universal parametrization of thermal photon rates in hadronic matter, Phys. Rev. C 91, 027902 (2015).

[34] O. Linnyk, V. Konchakovski, T. Steinert, W. Cassing, and E. L. Bratkovskaya, Hadronic and partonic sources of direct photons in relativistic heavy-ion collisions, Phys. Rev. C 92 , 054914 (2015).

[35] G. Basar, D. E. Kharzeev, and V. Skokov, Conformal Anomaly as a Source of Soft Photons in Heavy Ion Collisions, Phys. Rev. Lett. 109, 202303 (2012).

[36] G. Basar, D. E. Kharzeev, and E. V. Shuryak, Magnetosonoluminescence and its signatures in photon and dilepton production in relativistic heavy ion collisions, Phys. Rev. C 90, 014905 (2014).

[37] B. Muller, S.-Y. Wu, and D.-L. Yang, Elliptic flow from thermal photons with magnetic field in holography, Phys. Rev. D 89, 026013 (2014).

[38] A. Ayala, J. D. Castano-Yepes, C. A. Dominguez, L. A. Hernandez, S. Hernandez-Ortiz, and M. E. TejedaYeomans, Prompt photon yield and elliptic flow from gluon fusion induced by magnetic fields in relativistic heavy-ion collisions, Phys. Rev. D 96, 014023 (2017); Erratum, Phys. Rev. D 96, 119901(E) (2017).

[39] V. V. Goloviznin, A. V. Nikolskii, A. M. Snigirev, and G. M. Zinovjev, Probing a confinement by direct photons and dileptons, arXiv:1804.00559.

[40] A. Adare et al. (PHENIX Collaboration), Low-momentum direct photon measurement in $\mathrm{Cu}+\mathrm{Cu}$ collisions at $\sqrt{s_{N N}}=200 \mathrm{GeV}$, Phys. Rev. C 98, 054902 (2018).

[41] M. Allen et al. (PHENIX Collaboration), PHENIX inner detectors, Nucl. Instrum. Methods Phys. Res., Sect. A 499, 549 (2003).

[42] W. Anderson et al., Design, construction, operation and performance of a hadron blind detector for the PHENIX experiment, Nucl. Instrum. Methods Phys. Res., Sect. A 646, 35 (2011).

[43] K. Adcox et al. (PHENIX Collaboration), PHENIX central arm tracking detectors, Nucl. Instrum. Methods Phys. Res., Sect. A 499, 489 (2003).

[44] M. Aizawa et al. (PHENIX Collaboration), PHENIX central arm particle ID detectors, Nucl. Instrum. Methods Phys. Res., Sect. A 499, 508 (2003).

[45] L. Aphecetche et al. (PHENIX Collaboration), PHENIX calorimeter, Nucl. Instrum. Methods Phys. Res., Sect. A 499, 521 (2003).

[46] A. Adare et al. (PHENIX Collaboration), Evolution of $\pi^{0}$ Suppression in $\mathrm{Au}+\mathrm{Au}$ Collisions from $\sqrt{s_{N N}}=39$ to $200 \mathrm{GeV}$, Phys. Rev. Lett. 109, 152301 (2012).

[47] A. Adare et al. (PHENIX Collaboration), Neutral pion production with respect to centrality and reaction plane in $\mathrm{Au}+\mathrm{Au}$ collisions at $\sqrt{s_{N N}}=200 \mathrm{GeV}$, Phys. Rev. C 87, 034911 (2013).

[48] A. L. S. Angelis et al. (CERN-Columbia-OxfordRockefeller and CCOR Collaborations), Search for direct single photon production at large $p_{T}$ in proton proton collisions at $\sqrt{s}=62.4 \mathrm{GeV}$, Phys. Lett. 94B, 106 (1980). 
[49] A. L.S. Angelis et al. (CMOR Collaboration), Direct photon production at the CERN ISR, Nucl. Phys. B327, 541 (1989).

[50] T. Akesson et al. (Axial Field Spectrometer Collaboration), High $p_{T} \gamma$ and $\pi^{0}$ production, inclusive and with a recoil hadronic jet, in $p p$ collisions at $\sqrt{s}=63 \mathrm{GeV}$, Yad. Fiz. 51, 1314 (1990) [Sov. J. Nucl. Phys. 51, 836 (1990)].

[51] J. F. Paquet (private communication).

[52] A. Adare et al. (PHENIX Collaboration), Transverse energy production and charged-particle multiplicity at midrapidity in various systems from $\sqrt{s_{N N}}=7.7$ to $200 \mathrm{GeV}$, Phys. Rev. C 93, 024901 (2016).

[53] K. Aamodt et al. (ALICE Collaboration), Centrality Dependence of the Charged-Particle Multiplicity Density at Mid-Rapidity in Pb-Pb Collisions at $\sqrt{s_{N N}}=2760 \mathrm{GeV}$, Phys. Rev. Lett. 106, 032301 (2011).

[54] P. Aurenche, J. Ph. Guillet, E. Pilon, M. Werlen, and M. Fontannaz, A new critical study of photon production in hadronic collisions, Phys. Rev. D 73, 094007 (2006).
[55] C. Patrignani et al. (Particle Data Group Collaboration), Review of particle physics, Chin. Phys. C 40, 100001 (2016).

[56] The fit function to $p+p$ data originally used in Refs. [7,8] was updated in Ref. [40]. The parameters are $a=6.74 \times 10^{-3}, b=2.1, c=-3.3$. Systematic uncertainties also include possible shape variations at low $p_{T}$.

[57] When fitting the $0 \%-20 \% \mathrm{Au}+\mathrm{Au}$ data at $\sqrt{s_{N N}}=$ $200 \mathrm{GeV}$ over the range 1.0 to $2.0 \mathrm{GeV} / c$, which overlaps the range 0.9 to $2.1 \mathrm{GeV} / c$ used by ALICE, instead of the original range of 0.6 to $2 \mathrm{GeV} / c$ deployed by PHENIX, we obtain an inverse slope of $279 \pm 32 \pm 10 \mathrm{MeV} / c$. This value is consistent with the value $297 \pm 12 \pm 41 \mathrm{MeV} / c$ published by ALICE for the same centrality class for $\mathrm{Pb}+\mathrm{Pb}$ at $\sqrt{s_{N N}}=2760 \mathrm{GeV}$.

[58] E. L. Feinberg, Direct production of photons and dileptons in thermodynamical models of multiple hadron production, Nuovo Cimento A 34, 391 (1976). 